\title{
Value-oriented concept selection in aero-engine sub-systems design: the EVOKE approach
}

\author{
Marco Bertonia,b, Alessandro Bertoni ${ }^{b}$, Ola Isaksson', Henrik Amnellc, Christian \\ Johansson ${ }^{\mathrm{b}}$ \\ aDepartment of Mechanical Engineering, Blekinge Institute of Technology \\ (marco.bertoni@bth.se)
bProduct Innovation, Luleå University of Technology (marco.bertoni@ltu.se, alessandro.bertoni@ltu.se) \\ ‘GKN Aerospace Engine Systems Sweden (ola.isaksson@gknaerospace.com; \\ henrik.amnell@gknaerospace.com)
}

Copyright @ 2013 by Marco Bertoni. Published and used by INCOSE with permission.

\begin{abstract}
Value Driven Design methodologies extend the Requirements Management and Systems Engineering processes to reduce time and costs needed to identify the right solution direction to be pursued in detailed design. Emerging from the findings of an EU FP7 research project, the paper describes an approach, named EVOKE, that uses value as a basis for preliminary concept selection in the design of system components. EVOKE takes as input a list of value dimensions and drivers communicated by the system integrators, together with information about the high-level engineering characteristics of a candidate design, to enable early stage value analysis to be executed the by sub-system manufacturers. The approach and its technological enablers are described in detail through the use of a case study related to the design of new intermediate compressor case for turbofan engines.
\end{abstract}

\section{Introduction}

Aerospace industries are becoming similar to automakers in the way they deal with customer value. Operational cost is still the main success factor for a new aircraft development program, but dimensions such as comfort, timeliness, entertainment and environmental consciousness are becoming critical to attract passengers and eventually airlines. For instance, the Boeing 787 is marketed as "passenger-friendly" and aims at improving passenger experience thanks to better air quality and higher cabin pressure. Similarly, the AIRBUS 350 XWB has been designed with passenger wellbeing at the forefront, offering wider panoramic windows, larger overhead storage space, improved purity and humidity of cabin air and more precise temperature control zones.

Such features require radical changes at aircraft level. For instance, providing entertainment onboard means feeding the cabin with more electricity. The additional electrical power to be extracted from the engine turns into the need of redesigning engine components to host larger generators mounted on the core of the engine. This raises the level of integration required between the different parts of the system, which in turn asks for design activities to be better coordinated across suppliers since a very early phase, to deliver a unique product. This is 
particularly challenging nowadays, because development activities in the commercial aerospace sector are often conducted in large consortia, featuring hundreds of work packages and a wide network of sub-contractors.

Contractual requirements, signed off between directly interfacing partners, are the main reference for any work at any level of the aerospace supply network. While ensuring consistency they also introduce delays, because they are shared within the consortium only when mature enough to end up in contracts, often many months after the development work at aircraft level is initiated. In turn, they are made available to subcontractors with even more delay, which poses a problem to those manufacturers dealing with long development lead-time items. In order to ensure deliveries within the project timeframe, sub-system manufacturers initiate development activities on the basis of forecasts and market trends, with the risk of wasting time and resources in the wrong direction. This problem could be addressed by exchanging information on the intended use of the forthcoming solutions in a very preliminary design phase, establishing mechanisms to enable design loops to be executed before requirements are made available.

Targeting this industrial need, the European Commission's Seventh Framework (FP7) "Collaborative and Robust Engineering using Simulation Capability Enabling Next Design Optimisation" (CRESCENDO, http://www.crescendo-fp7.eu/) project has proposed an approach that uses "value dimensions" and "value drivers" to enable such feedback loops to be executed in the preliminary design stages of a commercial aerospace product.

This paper takes the perspective of an aero-engine sub-system manufacturer and describes an approach for preliminary design concept selection named "Early Value Oriented design exploration with KnowledgE maturity" (EVOKE). EVOKE takes as input the value dimensions and drivers communicated by the system integrators, and uses them to rank early design concepts using value as metrics. The paper initially summarizes the Value Driven Design methodology developed within CRESCENDO (Isaksson et al. 2013), highlighting its distinguishing features. Then it describes and exemplifies EVOKE in a case study related to the development of an aero-engine intermediate compressor case (IMC) technology. A discussion section follows, reporting on implementation and validation issues.

\section{Context}

CRESCENDO proposes a methodology complementing traditional Systems Engineering (SE) (Systems Engineering Handbook, 2010) processes that uses the concept of value for early design concept generation and selection (Figure 1). The methodology is inspired by previous Value Driven Design (VDD) approaches proposed in literature (Castagne et al. 2009, Collopy and Hollingsworth 2011), and enables what-if assessment loops to be executed at all levels of the supply chain in the very early stage of the aircraft design process, before detailed requirements are made available by the aircraft manufacturer.

The Value Creation Strategy (VCS) is the entity (or the document) that enables the sharing of preliminary design information among the parties in the supply chain. A VCS carries the description of a specific context, which initially includes a set of rank-weighted needs for the aircraft and (later) for the aero-engine, and that it is further detailed in value dimensions and value drivers. Rank-weighted needs emerge from the analysis of passengers, customers and stakeholders expectations, which are typically collected via focus group meetings, individual face-to-face interviews or surveys. Expectations are complemented and interpreted in the light of available information on airlines, market segments, regulatory requirements and lifecycle considerations. Each need is then cascaded down to a single value dimension, which is detailed in several value drivers, which express value-adding engineering characteristics for a specific problem context.

The underlying hypothesis of the proposed methodology is that the VCS allows each 
manufacturer to reach a more advanced stage in the development of its components compared to what happens when only requirements are made available. Value Drivers (VDs) indicate solution directions that are acknowledged to influence the customer and end user perceived value in a given context. Although less formalized and more volatile than the requirements description, these dimensions carry contextual information that is neither formalized nor shared by the aircraft manufacturer with the collaborating partners, and that can support decisions makers in early trade-off decisions. Depending on the complexity of the project and from the supply network structure, the VCS can feature one or more iterations.

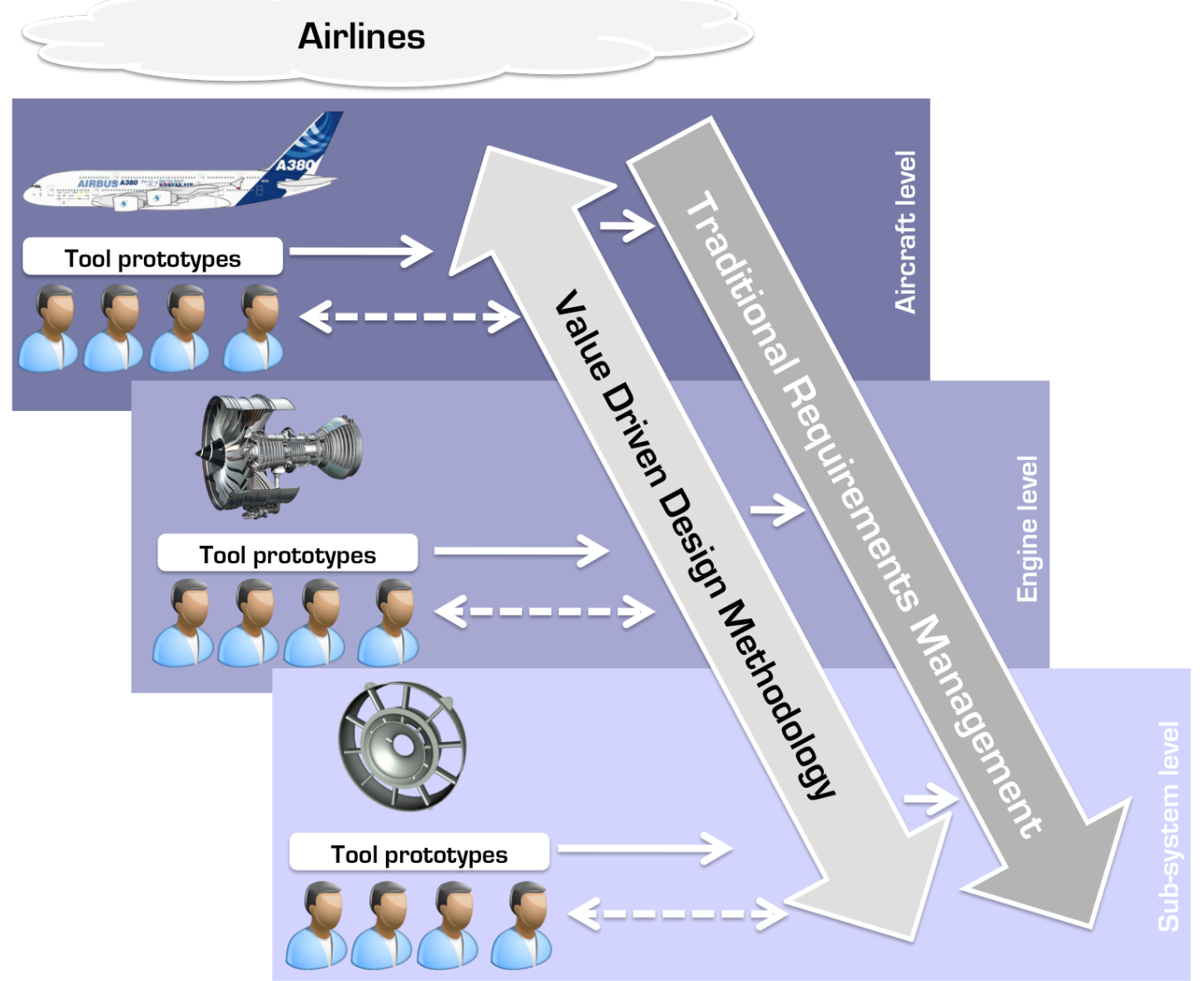

Figure 1. The CRESCENDO Value Driven Design Methodology (Isaksson et al. 2013)

Moving from the description of value dimensions and drivers, the design team sketches alternative solution directions. These early design concepts are specified in terms of Quantified Objectives (QOs), which describe, from a more engineering point of view, how to satisfy a list of needs. In the later iterations, QOs become part of the VCS, and are communicated back to the system integrators to influence architectural decisions. They are also used locally to establish a first list of requirements for the solution.

\section{EVOKE process description and application example}

The VCS is essentially the metrics against which the sub-system manufacturer can evaluate what to develop, by assessing how a solution concept contributes to engine and aircraft value in a more structured way than basing the decision on experience and forecasts.

What should be the measure of such "contribution", and how it should be measured, is an open problem in literature (Soban et al. 2011). Value assessment requires an intuitive and 
meaningful value function that enables direct comparison among alternatives (Collopy and Hollingsworth 2011). Monetary units, such as dollars, are then used as convenient, practical, and universally understood metrics for value (see; Hazelrigg 1997, Collopy and Hollingsworth 2011). However, VDD is also a fundamentally social activity, whose effectiveness is strongly related to the phenomena of group dynamics and sensemaking (Collopy 2012). Engineers discuss value both within their sub-system and with other sub-systems, while program managers communicate amongst themselves, with their design teams and with other stakeholders. Monetizing value attributes is often cumbersome and meaningless in an early phase, and can potentially impede the timely communication of critical information between all these actors (Soban et al. 2011). This asks for the development of a value function that is more qualitative and that focuses both on physical/functional and relationship-based aspects (Soban et al. 2011).

Emerging from this gap, the authors have developed a structured method that use the information contained in the VCS to perform a qualitative value assessment and, eventually, to rank design alternatives on the basis of their "goodness" at system level (Figure 2).

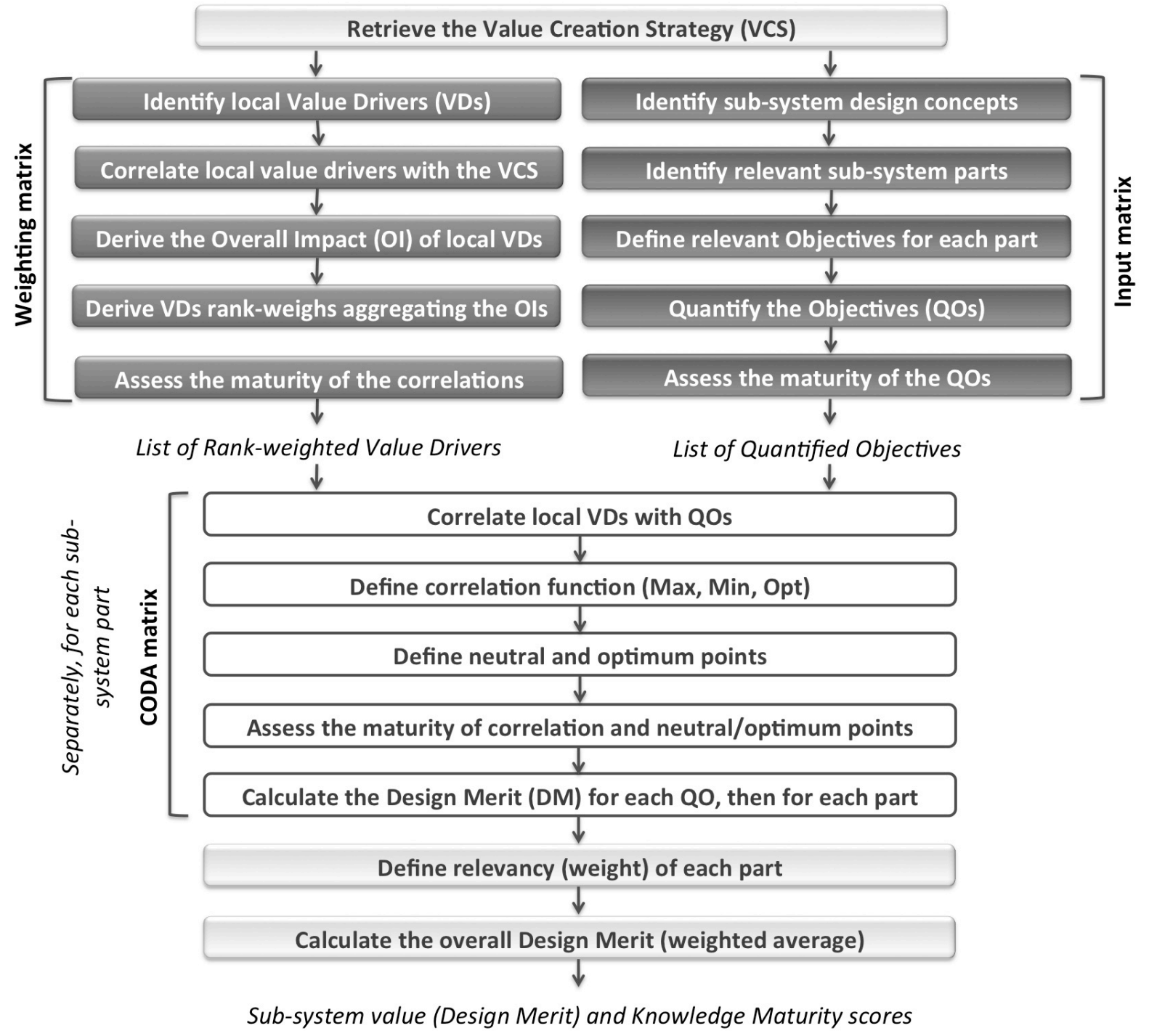

Figure 2. The EVOKE process

EVOKE is based on 3 matrixes: the Weighting Matrix, which cascades down the system-level VCS to local (sub-system) VDs, the Input Matrix which gathers information about the characteristics of each design alternative under consideration, and the Customer Oriented 
Design Analysis (CODA) matrix (Woolley et al. 2001), which renders a Design Merit (DM) for each design. These matrixes are complemented by a method that provides a feedback to the designers about the reliability of the value analysis results, which is about the Knowledge Maturity on which the value models are built.
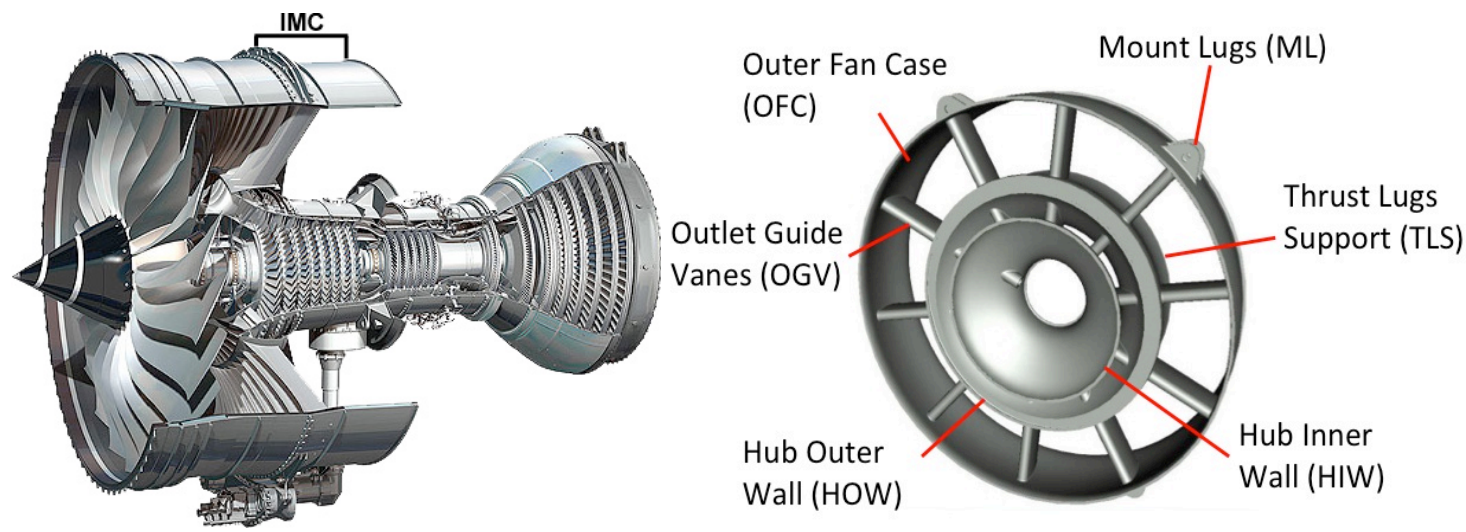

Figure 3. Intermediate Case (IMC) position in a Rolls Royce Trent XWB engine

EVOKE has been exemplified and demonstrated in a case study related to the development of an aero-engine intermediate compressor case (IMC) (Figure 3). The IMC supports surrounding parts, keeps the airflows separated, off-takes bleed air and transfers the thrust from the engine to the airframe.

\section{Weighting matrix}

Development activities at sub-system level are initiated when the VCS is retrieved from the aircraft and engine manufacturers. As previously explained, the VCS is composed of rank-weighted value dimensions and related VDs. For simplicity purposes, in this example it features only 7 value dimension at engine level, which refer to 7 major needs: Emissions Levels, Specific Fuel Consumption, Noise Level, Operational Reliability, Bleed Air Quality, Direct Operating Costs and Electrical Power Output.

After retrieving the VCS, the design team defines sub-system VDs. Emerging from the engine architecture preliminary description, and from generic sub-system value dimensions of interest (such as Long Term Profitability or Risk), the work has brought to the definition of 10 different drivers for the IMC:

- Temperature, Pressure, Weight and Drag refer to engineering dimensions that affect the overall system behavior;

- Reliability, Availability, Adaptability, Manufacturability and Weldability capture aspects related to the manufacturing, operational and maintenance phase;

- Knowledge Reuse, which is more intangible, summarizes the risk related to the development of a new component or technology.

These drivers are identified by the design team in the very early beginning of the value assessment process, and represent the main criteria upon which the value of an IMC concept will be judged. Ten was considered as a good trade-off between simplicity and detail, allowing the drivers to be managed without being overwhelmed with too many details.

The weights in the value assessment matrix (i.e., the importance of the VDs in terms of value fulfillment) depend from the VCS. Different VCSs emphasize different needs and, in turn, aspects of the local solution that play a stronger role in the fulfillment of customer expectations. Figure 4 shows 2 different VCS, one performance-oriented, and one 
comfort-oriented, as emerged from the case study. The first emphasizes dimensions such as Emissions Levels, Specific Fuel Consumption and Direct Operating Costs. The second focuses on Bleed Air Quality and Electrical Power Output. These strategies influence the relative importance of the sub-system VDs, as shown in the right end side of Figure 4. Temperature, Pressure and Weight are less significant from a value perspective if the second VCS applies to the problem. At the same time, Drag, Adaptability and Weldability are emphasized, as more comfort is achieved also by being able to adapt the sub-system to different operating conditions (e.g., through upgrading).

- Performance-oriented VCS

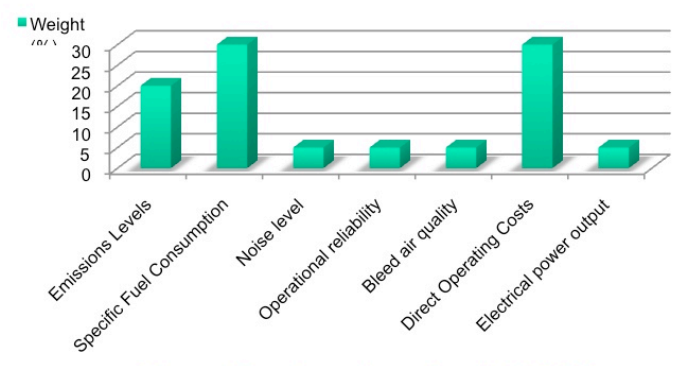

- Comfort-oriented VCS

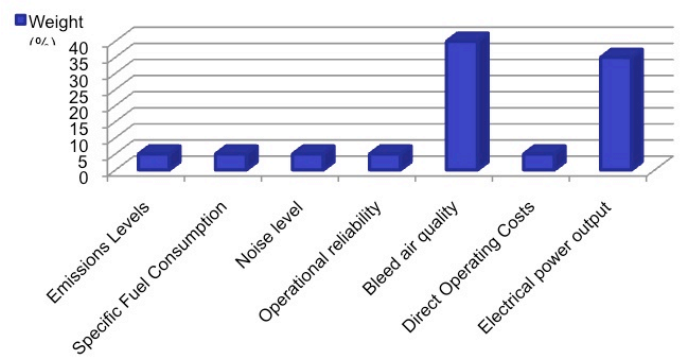

Relative importance of the Value Drivers

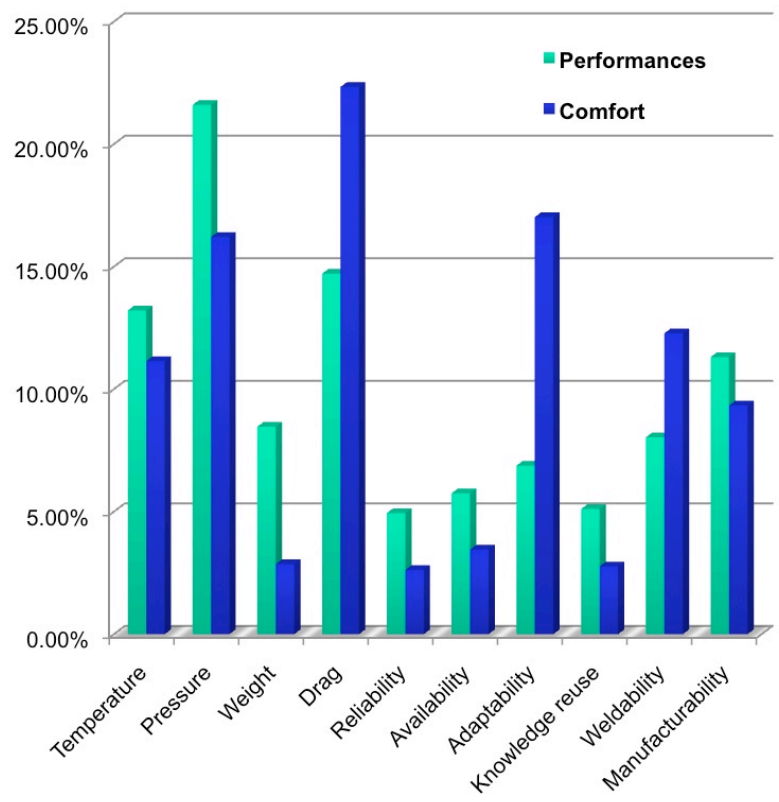

Figure 4. VCS impact on sub-system VD weights

EVOKE uses a Weighing matrix that renders a set of Normalized weights to link the VCS to the sub-system VDs. Weights are obtained by coupling engine and local (sub-system) VDs as shown in Figure 5. A local VD can feature a strong, medium or weak correlation with a VD at engine level. These correlations are modeled using (0.9), (0.3) and (0.1) coefficients. For instance, Temperature and CO2 Emissions are strongly correlated, because it is assumed that $\mathrm{C}^{2}$ production is significantly influenced by temperature changes in the IMC (therefore a 0.9 correlation is set). Temperature and Particulates feature a medium correlation, because the production of pollutants varies only within a restricted range due changes in temperature (therefore a 0.3 correlation is set). Eventually, Weight and Particulate feature a low correlation (i.e., 0.1), because modifying the weight of the IMC will have a very minor effect on the production of particulate (e.g., a very small reduction due to a slightly lighter engine).

For a given need, Correlations coefficients are summed along each sub-system VD, to first obtain a Sum of Correlations. This process is repeated for all the sub-system VDs. The Sum for a driver (such as for Temperature) is weighted against the Sums for all the other drivers, to obtain the relative importance of the driver for a given need. Furthermore, this weighted Sum is multiplied for engine value dimension weight, to obtain an Overall Impact score. These score reflect the importance of the relationship between an engine need and a sub-system VD within the entire set of needs provided. This operation eventually causes different VCSs to render different aggregated scores, and therefore to influence the way the total Design Merit for a solution is computed in the CODA matrix. 


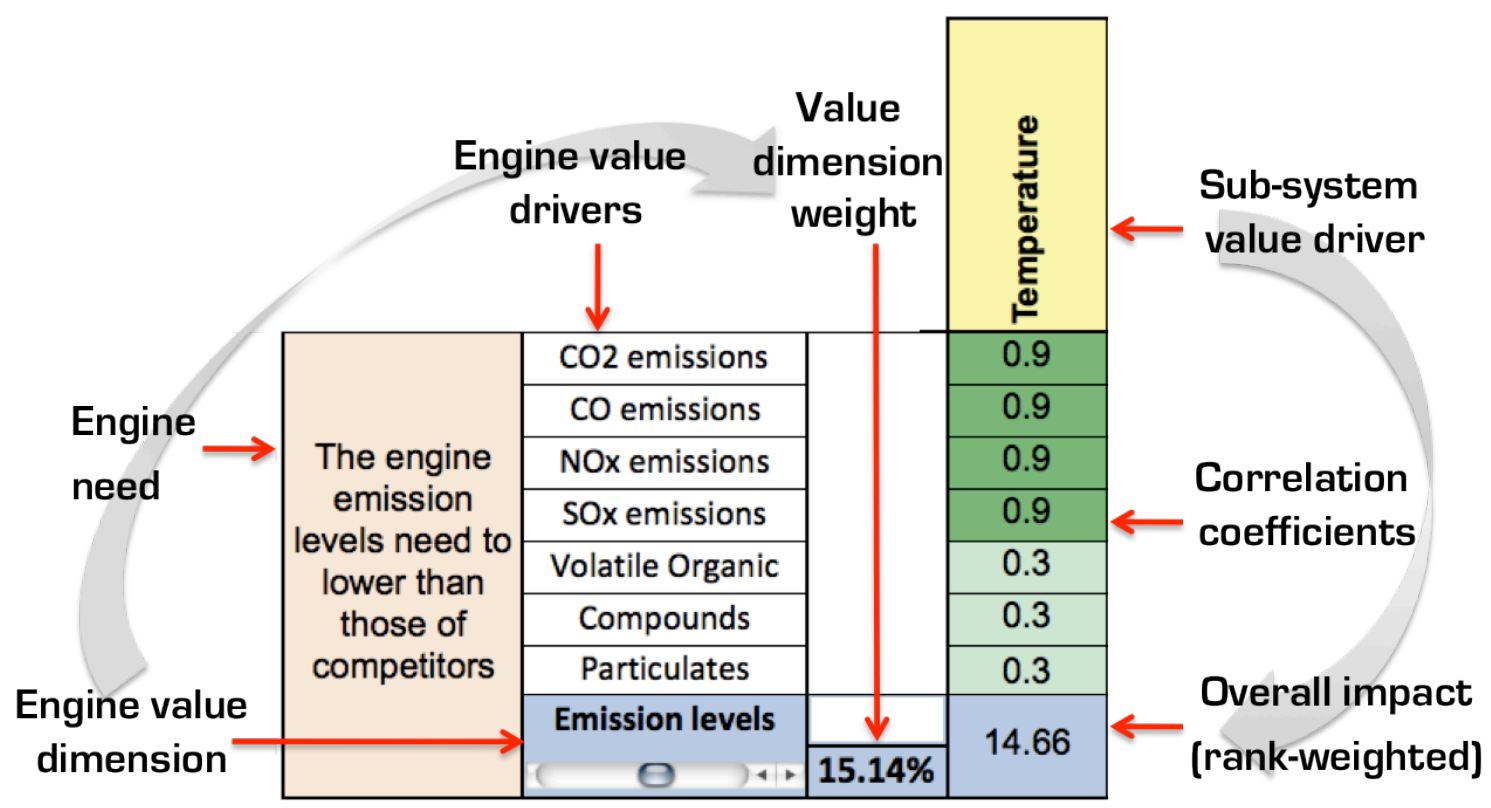

Figure 5. Extract from the weighting matrix (Emissions Levels vs. Temperature)

The list of weighted sub-system VDs is the first inputs of the CODA matrix for value assessment.

\section{Input matrix}

The assessment process requires the design team to define the high-level features of sub-system alternatives that will be later benchmarked using the CODA matrix. For simplicity purposes, the analysis has been reduced to 2 main alternative concepts, Option \#1 and Option \#2, which differ mainly in term of manufacturing technology and implementation of a bleed-air off-take function.

- Option \#1 embodies a traditional design, exemplified by a fully casted IMC featuring 8-10 inner struts and 16-20 outer struts. It includes also a casted hub that implements a bleed off-take function.

- Option \#2 features a more radical design, characterized by an increased use of composite material. This design features a fabricated Hub that, for safety reasons, does not implement a bleed air off-take function.

The empirical investigation has shown that the sub-system manufacturer is more interested to understand how much a solution is better-or worse - than existing options, than to obtain an absolute value score. Hence, the value assessment needs to be supported by solid benchmarks to enable more effective decision-making. The manufacturer expressed a preference toward using a product Baseline (reflecting the value of an existing solution) and a Target (reflecting a vision emerging long-term forecasts, calculated in this example as $110 \%$ of the baseline value contribution) as references for the value study. To describe in detail these 4 options, the IMC has been divided into 6 main parts, as shown in Figure 3: Mount Lugs (ML), Outer Fan Case (OFC), Outlet Guide Vanes (OGV), Thrust Lugs Support (TLS), Hub Outer Wall (HOW) and Hub Inner Wall (HIW). These parts have been detailed with information about geometry, shape, material, reuse of technology, production lead-time and accessibility to experts (typically with different levels of confidence, see the Knowledge Maturity section). Once quantified, these inputs were named Quantified Objectives (QOs) (Table 1). 
Table 1: Extract from the Input Matrix (Mount Lugs)

\begin{tabular}{|c|c|c|c|c|c|c|}
\hline Quantified objectives & Units & Baseline & Option \#1 & Option \#2 & $\begin{array}{c}\text { Upper } \\
\text { limit }\end{array}$ & $\begin{array}{c}\text { Lower } \\
\text { limit }\end{array}$ \\
\hline Mount Lugs & & $\begin{array}{l}\text { Aluminum alloy } \\
\text { engine mount }\end{array}$ & $\begin{array}{c}\text { Titanium bolt } \\
\text { engine mount, } \\
\text { which introduces } \\
\text { a weight penalty } \\
\text { on the component } \\
\text { compared to the } \\
\text { baseline. }\end{array}$ & $\begin{array}{c}\text { Titanium bolt } \\
\text { engine mount, } \\
\text { which introduces } \\
\text { a weight penalty } \\
\text { on the component } \\
\text { compared to the } \\
\text { baseline. }\end{array}$ & & \\
\hline •Thickness & $\mathrm{mm}$ & 45 & 45 & 50 & 80 & 30 \\
\hline -Reuse of technology & $\%$ & $65.00 \%$ & $65.00 \%$ & $60.00 \%$ & $80.00 \%$ & $25.00 \%$ \\
\hline $\begin{array}{l}\text {-Housing for additional } \\
\text { components/functions }\end{array}$ & $\mathrm{m}^{\wedge} 3$ & 0.3 & 0.3 & 0.35 & 0.8 & 0.2 \\
\hline -Access to experts & $\%$ & $65.00 \%$ & $65.00 \%$ & $60.00 \%$ & $75.00 \%$ & $45.00 \%$ \\
\hline -Production lead time & $\mathrm{h}$ & 18 & 18 & 23 & 30 & 15 \\
\hline -Line commonality & $\%$ & $50.00 \%$ & $50.00 \%$ & $49.00 \%$ & $70.00 \%$ & $10.00 \%$ \\
\hline
\end{tabular}

Upper and lower limits (from historical series of data) are also elicited to enable the value assessment calculation in the following step. The list of QOs for each part of each sub-system alternative is the second inputs for the CODA-based value assessment matrix.

\section{CODA-based value assessment matrix}

EVOKE adapts uses the Customer Oriented Design Analysis (CODA) matrix to map QOs against VDs, and to obtain an overall DM score for each part of the system. The scores are later aggregated to obtain the overall sub-system value. The CODA approach is an extension of Quality Function Deployment (QFD) (Akao and Mizuno 1994) and uses non-linear optimization type functions (positive or negative) to map needs to engineering characteristics. This is believed to better approximate the customer response to changes in a product attribute (Andersson and Sullivan 1993). Table 2 shows an extract of the CODA matrix used for the evaluation of the IMC alternatives.

Table 2: Extract from the CODA matrix

\begin{tabular}{|c|c|c|c|c|c|c|}
\hline & & \multicolumn{5}{|c|}{ Surface finishing } \\
\hline & & $\begin{array}{l}\text { Design } \\
\text { parameter }\end{array}$ & 0.11 & $\begin{array}{l}\text { Constraints } \\
\text { (Upper and } \\
\text { Lower) }\end{array}$ & 0.15 & 0.01 \\
\hline Value drivers & $\begin{array}{l}\text { Normalised } \\
\text { weights }\end{array}$ & Correlation & $\begin{array}{c}\text { Neutral point } \\
\text { or optimum } \\
\text { point }\end{array}$ & $\begin{array}{c}\text { Relationship } \\
\text { Type } \\
\text { (Min, Max, Opt) }\end{array}$ & $\begin{array}{c}\text { Tolerance } \\
\text { (for type Opt) }\end{array}$ & $\begin{array}{c}\text { Design } \\
\text { Merit }\end{array}$ \\
\hline Drag & $17.54 \%$ & 0.90 & 0.08 & Min & & $39.60 \%$ \\
\hline Knowledge reuse & $3.89 \%$ & 0.10 & 0.03 & Max & & $92.13 \%$ \\
\hline Manufacturability & $13.22 \%$ & 0.30 & 0.08 & Max & & $61.44 \%$ \\
\hline
\end{tabular}

As first task, the design team defines the correlation between a QO and a VD, which can be strong (0.9), medium (0.3) and weak (0.1). The selection of these coefficients is discussed further in the Sensitivity Analysis section. In the IMC example Surface finishing (which is expressed as friction coefficient) features a strong correlation (0.9) with Drag, a medium correlation (0.3) with Manufacturability and a weak correlation (0.1) with Knowledge Reuse. Correlations express the extent to which a VD is positively or negatively impacted by a change in the value of the QOs. This impact is further modeled by a Relationship Type (Maximize, Minimize, Optimize). In the example, Drag is improved when the friction coefficient is minimized, while Manufacturability and Knowledge Reuse when is maximized 
(because a better surface finishing increases production lead time and requires an higher level of expertise to be executed). For some QOs, an optimizing function offers a more viable alternative than minimization and maximization.
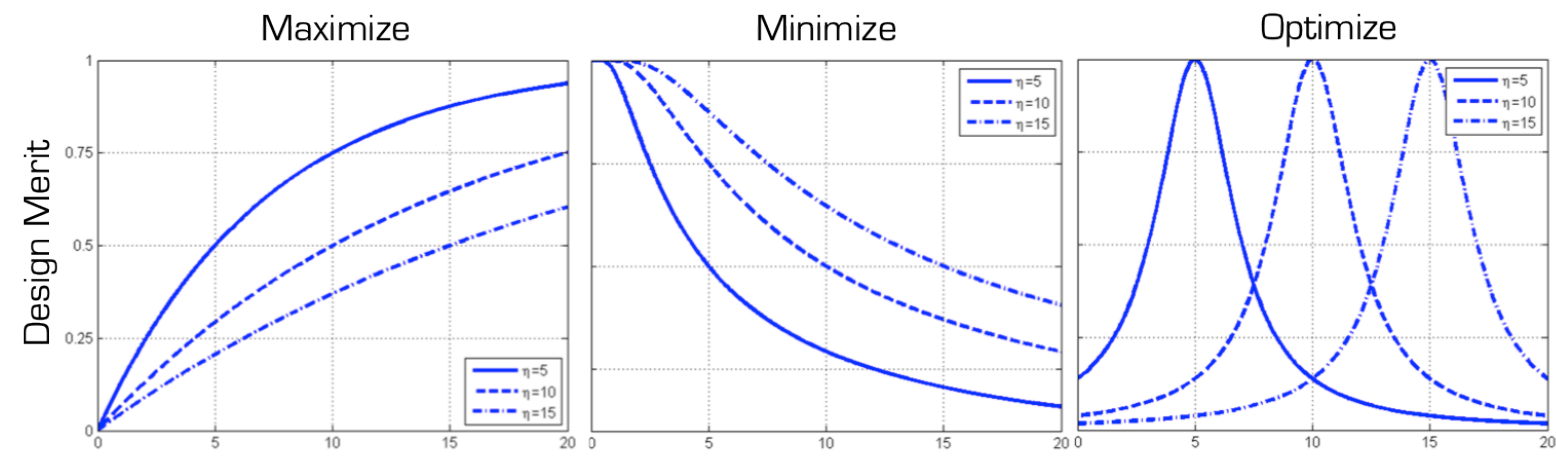

Parameter value, $\rho$

Figure 6. Relationship types in the CODA matrix

A main assumption of CODA is that the degree of need fulfillment is not linear but exponential, to reflect the diminishing returns that are typical in utility curves. Figure 6 shows the values rendered by the Max, Min and $O p t$ functions while varying the value of a QO $(\rho)$ and its neutral (or optimum) point $(\eta)$. In maximization and minimization type functions the $\mathrm{DM}$ assumes a value of $50 \%$ when a $\mathrm{QO}$ is equal to the neutral point value. In optimization type function DM assumes the optimum value $100 \%$ when the QO is equal to the neutral point value. Once DM scores are obtained for all parts, they are aggregated to obtain a Total Design Merit (TDM). Scores are aggregated by means of a weighted sum. Weights are obtained from the Weighting Matrix, as described in one of the previous section.

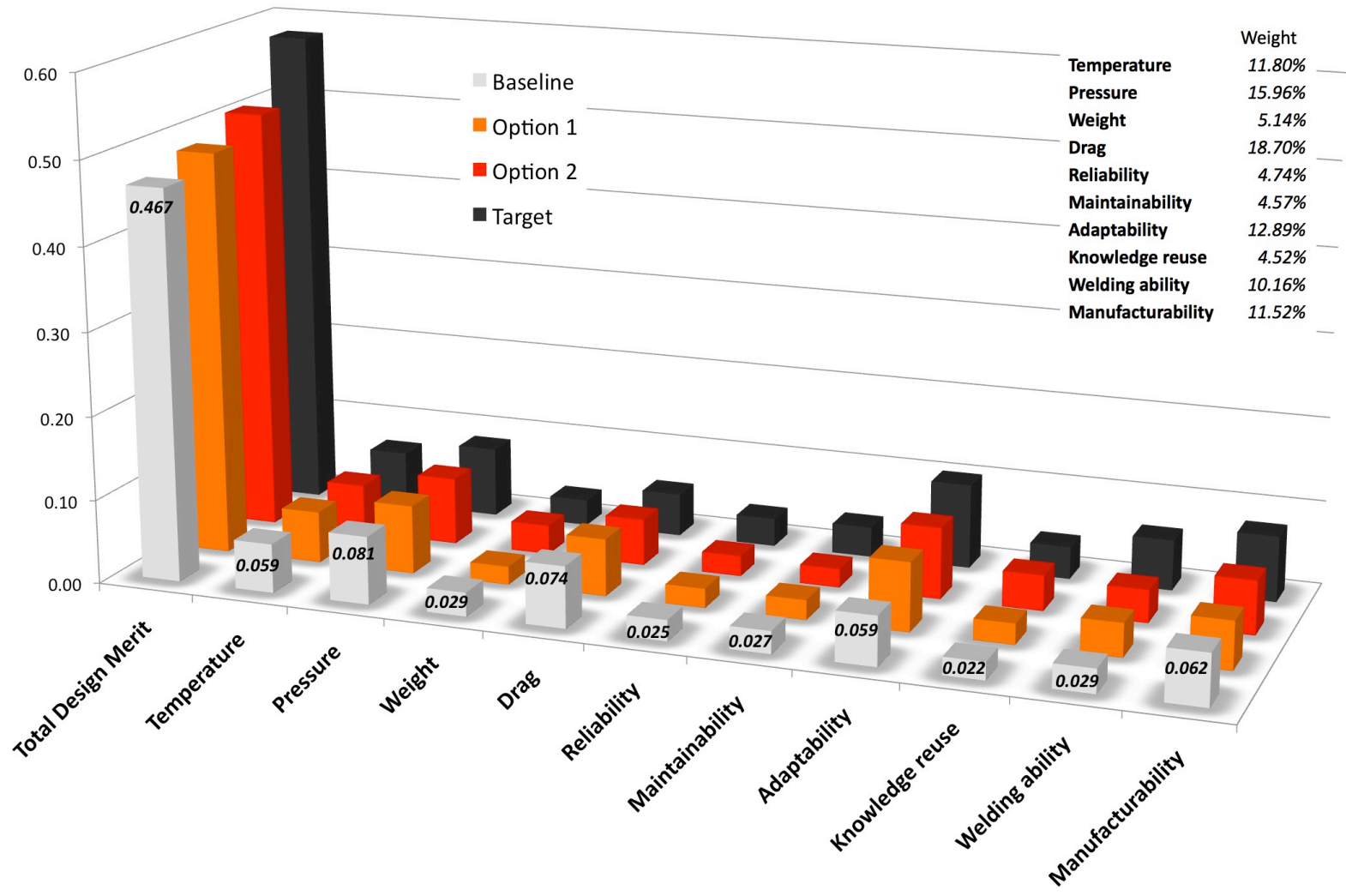

Figure 7. Results of the CODA matrix 
Figure 7 shows the TDM for all four options, detailing the value contribution for each VD (already considering the VD weight). Although Option \#2 renders the highest Total Design Merit, topping 50,68\%, it does not yet meet the Target set for this design activity. The team has to decide if to invest resources in optimizing this design, to reject it, or to approve it for further development. The information displayed in Figure 7 also highlights drivers that necessitate deeper value studies, as well as areas where to trade off excellent performances with others where value contribution is lower.

\section{Knowledge Maturity assessment}

Value models attempt to quantify a generally subjective and qualitative arena, namely making preliminary design decisions with relatively little information. There is a risk associated with such early phase "quantification" of choices, because the matrixes used in EVOKE are to large extent built on assumptions and populated with figures characterized by different levels of confidence (i.e., for known technologies the input data can be widely known and assessed, for radically innovative solutions they might just be an educated guess). The use of value models has the potential to effectively devalue the engineering process itself by giving the appearance that engineering is occurring. Hence, the intrinsic subjectivity and qualitative nature of the process have to be addressed and communicated to correctly support decision-making (Blanco et al. 2007). Uncertainty needs to be handled by assisting the decision makers in achieving a better understanding of what these uncertainties, ambiguities, and assumptions actually involve, which is the level of maturity (Grebici et al. 2007) of the knowledge on which the value model is built.

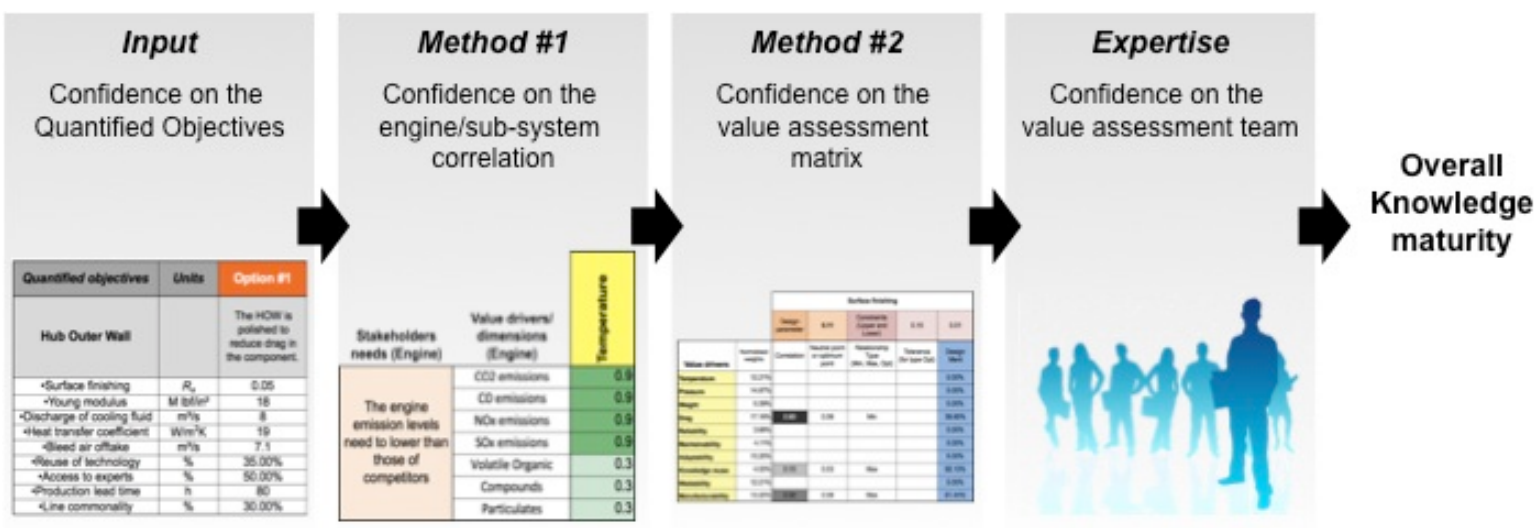

Figure 8. Knowledge Maturity assessment high-level process

Bohn (1994) defines maturity of knowledge as "understanding the effects of the input variables on the output" (ibid, p.63). Emerging from this definition, EVOKE proposes an approach for Knowledge Maturity (KM) assessment, which answers questions such as:

- Does the output of the value model reflect assumptions or verified facts?

- Are there specific knowledge assets that would need further development to clarify the meaning a value score?

EVOKE computes Knowledge Maturity over three dimensions - Input, Method and Expertise (Figure 8), similarly to what proposed by Johansson et al. (2011).

The assessment process considers the following elements in the calculation:

- The QOs in the Input Matrix may both be derived from lab tests and statements from independent sources (high KM), or may simply reflect the opinions of the design team, without the support of factual data (low KM). This dichotomy is 
particularly evident when assessing the lifecycle behavior of the product: correlation coefficients might reflect what observed and reported from a number of system implementations, or might be derived by interpolation means.

- In the Weighting Matrix the design team might not have a clear perception of the correlations between VDs at engine and sub-system level. In some cases, they might mirror well-known mathematical functions (high KM); in other cases they might just reflect the opinion of experts (low KM).

- In the CODA Matrix, correlations, functions, neutral/optimum points and tolerances might reflect well-known phenomena, which are supported by experimental evidence and cases (high $\mathrm{KM}$ ), or might be just a structure of imagination with poor experimental evidence and rationale (low KM).

- Eventually, the design team might have a long and verified experience within all the disciplines/areas the VDs point to (high maturity), or only within a few of these areas (low maturity).

Knowledge Maturity is expressed by a single scalar, ranging from 1 (lowest maturity) to 5 (highest maturity), as proposed by Johansson et al. (2011). A Knowledge Maturity matrix collects such information (for each VD) to produce an overall maturity score for a design.

Figure 9 displays the value analysis outcomes together with the results of the KM assessment. A major issue in Figure 7 is that Baseline, Option \#1 and Option \#2 feature very similar DM scores; therefore decision makers do not have a clear perception of which alternative to pursue. Adding a KM perspective, they will likely prefer Option $\# 1$, because the value contribution of Option \#2 is based on less mature knowledge, and might be riskier to develop.

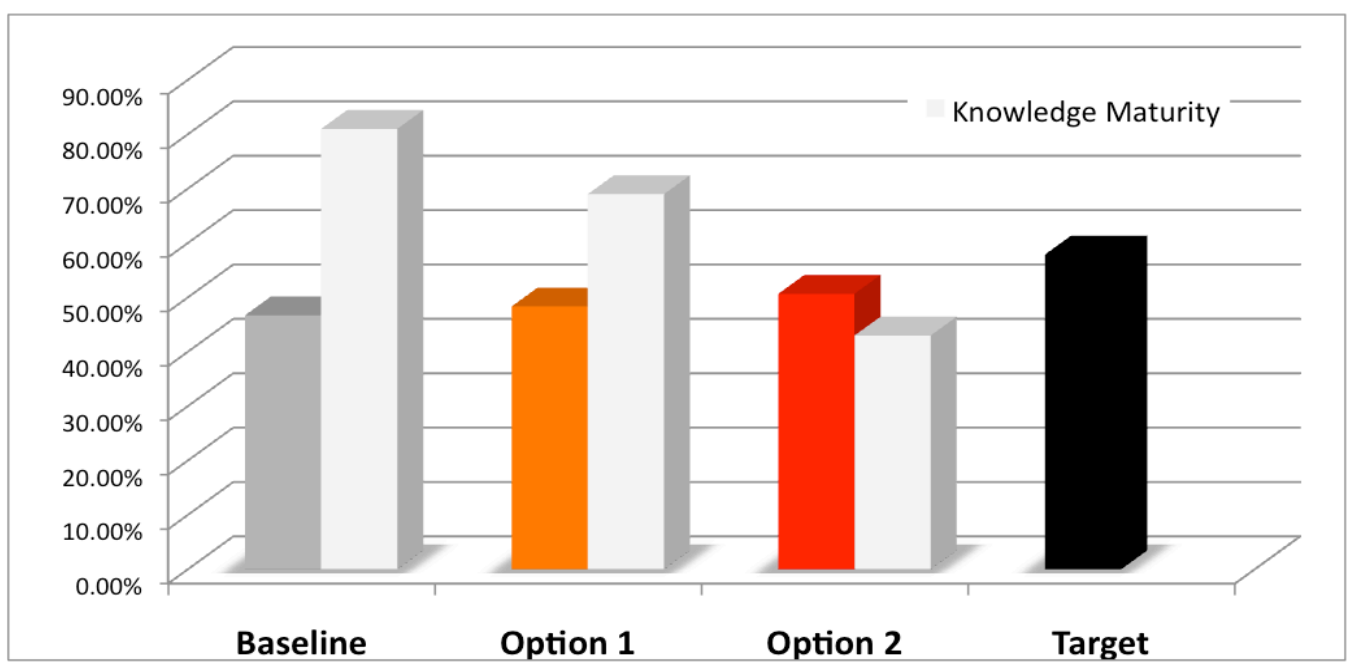

Figure 9. Value analysis results complemented by Knowledge Maturity

\section{Sensitivity analysis}

The 0.9-0.3-0.1 (low/medium/high) scale was considered a reasonable choice dealing with highly volatile data. A more granular scale would have given the impression that engineering (quantitative evaluation) was occurring, while the approach is mostly subjective and qualitative. Not only it would have increased complexity, but also it would have been more difficult to agree upon, requiring more time and effort to populate the value model. Rather, the purpose is to provide a tool for quick what-if analyses to be executed in an early stage.

EVOKE was tested for sensitivity following the approach proposed by Ghiya et al. (1999). Sensitivity can be defined as the probability of observing large changes in relative worth 
when switching rating scale or weighting method (Takai and Kalapurackal 2012). The authors have tested the effects of using different correlation scales in the Weighting and CODA Matrix, de-emphasizing the importance of the strong and weak relationships.

Table 4 shows the results of this analysis for a representative VCS. Overall, the ranking between the design alternatives is not impacted by the changes in the correlation scale. The Target option is always the most desirable, and is followed by Option \#2, Option \#1 and Baseline. The largest route mean square is recorded when the weights are changed from 0.9-0.3-0.1 to $0.9-0.3-0.0$, which is when the weak relationship is not considered in the model. In all the other cases the route mean square reaches a maximum of $1.02 \%$.

Table 3: Results of the EVOKE sensitivity analysis

\begin{tabular}{|c|l|l|l|l|}
\hline DESIGN MERIT & Baseline & Option 1 & Option 2 & Target \\
\hline $0.9-0.3-0.1$ & $48.01 \%$ & $48.85 \%$ & $49.41 \%$ & $58.01 \%$ \\
\hline $0.7-0.3-0.1$ & $47.83 \%$ & $48.59 \%$ & $49.19 \%$ & $57.83 \%$ \\
\hline $0.5-0.3-0.1$ & $47.56 \%$ & $48.23 \%$ & $48.86 \%$ & $57.56 \%$ \\
\hline $0.4-0.2-0.1$ & $47.57 \%$ & $48.33 \%$ & $49.37 \%$ & $57.57 \%$ \\
\hline $0.9-0.3-0.0$ & $46.29 \%$ & $46.85 \%$ & $47.46 \%$ & $56.29 \%$ \\
\hline
\end{tabular}

The same analysis was conducted in the extreme situation of setting the rank-weight of an engine value dimension to $100 \%$, while the others to $0 \%$. Even in this case the ranking is not impacted by the changes in the correlation scale (Table 5). The exception is the 0.9-0.3-0.0 scale, which renders a Baseline more value adding than Option \#1. The root mean square change analysis does not significantly differ from the previous. A small increase is perceivable (1.19\%) when using the 0.4-0.2-0.1 weights. Again, the 0.9-0.3-0.0 metric shows behavior not aligned with the others, underlying the inadequacy of these correlation weights.

Table 4: Results of the EVOKE sensitivity analysis (with $n-1$ value dimensions $=0 \%$ )

\begin{tabular}{|c|l|l|l|l|}
\hline DESIGN MERIT & Baseline & Option 1 & Option 2 & Target \\
\hline $0.9-0.3-0.1$ & $49.15 \%$ & $50.45 \%$ & $55.08 \%$ & $59.15 \%$ \\
\hline $0.7-0.3-0.1$ & $49.00 \%$ & $50.33 \%$ & $54.78 \%$ & $59.00 \%$ \\
\hline $0.5-0.3-0.1$ & $48.79 \%$ & $50.16 \%$ & $54.37 \%$ & $58.79 \%$ \\
\hline $0.4-0.2-0.1$ & $48.40 \%$ & $50.13 \%$ & $54.55 \%$ & $58.40 \%$ \\
\hline $0.9-0.3-0.0$ & $48.53 \%$ & $48.36 \%$ & $53.15 \%$ & $58.53 \%$ \\
\hline
\end{tabular}

\section{Conclusions}

EVOKE is an approach for preliminary concept selection that uses value as a basis for decision. It enables qualitative value analyses to be executed early on, before detailed requirements are made available, by taking as input the value dimensions and drivers 
communicated by the system integrators by means of the so called Value Creation Strategy, together with information about the high-level engineering characteristics of the sub-systems under consideration.

The feasibility of the approach was verified with the industrial partners. By means of live demonstrations, the authors have received qualitative feedback from engineers and project stakeholders about strengths and opportunities related to the implementation of EVOKE for aero-engine sub-system design. As main findings from these preliminary validation activities, the authors observed that the approach could enhance awareness on:

- The relative importance of the needs cascaded down from the system integrators, enabling the design teams to better identify the most important dimensions to prioritize at the beginning of the design activity, reducing rework later in the process.

- The relationship between system-level needs and the engineering characteristics of the specific component designed, mainly because non-linear merit functions are believed to better approximate the customer response to changes in product attribute compared to QFD or other approaches.

- The reliability of the value analysis, through the use of knowledge maturity assessment into the conceptual trade-off analysis.

Demonstration and validation activities are still in their early beginning and future work is planned to gather more factual data about the effectiveness of the approach in a range of design situations. This will be accompanied by further studies on the sensitivity of the EVOKE matrixes, as well as on the effects and benefits of knowledge maturity principles, which have not been scientifically proven yet.

The use of color-coded CAD models as a means to display the results of the value assessment activity has also been proposed to improve communication of value information within the design team. The approach has been implemented in a SIEMENS NX environment and tested both in industry and in design sessions with students (Bertoni et al. 2012). In the future, such testing activities will be extended, observing engineers and designers using the tool in real working situation. The research will also address the problem related the implementation of VDD methodologies in the extended enterprise, such as mechanisms and guidelines for the management of intellectual property rights (IPR) when exchanging value information.

\section{Acknowledgments}

The research leading to these results has received funding from the European Union Seventh Framework Programme (FP7/2007-2013) under grant agreement $n^{\circ} 234344$ (www.crescendo-fp7.eu/).

\section{References}

Akao, Y. and Mizuno, S., 1994. QFD: The customer-driven approach to quality planning and deployment. Asian Productivity Organization, Tokyo.

Anderson, E. W., Sullivan, M. W. 1993. "The antecedents and consequences of customer satisfaction for Firms." Marketing Science Vol. 12 (Spring): 125-143.

Bertoni, M., Bertoni, A., Isaksson, 0. 2012. "Experiences with Value Visualisation in preliminary design: results from an aero-engine component study." Proceedings of the $1^{\text {st }}$ International Conference on Through-life Engineering Services, Nov. 5th-6th, Cranfield, UK. 
Blanco, E., Grebici, K. and Rieu, D. 2007. "A unified framework to manage information maturity in design process." International Journal of Product Development 4(3): 255-279.

Bohn, R. E. 1994. "Measuring and managing technological knowledge." Sloan Management Review (Fall): 61-73.

Castagne, S., Curran, R., and Collopy, P. 2009. "Implementation of Value-Driven Optimisation for the Design of Aircraft Fuselage Panels." International Journal of Production Economics 117: 381-388.

Collopy P. D. and Hollingsworth P.M. 2011. "Value-Driven Design." Journal of Aircraft 48 (3): 749-759.

Collopy, P. 2012. "A Research Agenda for the Coming Renaissance in Systems Engineering." Proceedings of the 50th AIAA Aerospace Sciences Meeting Including the New Horizons Forum and Aerospace Exposition, 10p.

Ghiya, K. K, Bahill, A. T. and Chapman, W. 1999. “QFD: validating robustness.” Quality Engineering 11(4): 593-611.

Grebici, K., Goh, Y. M., Zhao, S., Blanco, E., and McMahon, C. 2007. "Information Maturity Approach for the Handling of Uncertainty within a Collaborative Design Team." Paper presented at the 11th International Conference on Computer Supported Cooperative Work in Design.

Hazelrigg, G., 1998. “A Framework for Decision-Based Engineering Design.” Journal of Mechanical Design, 120(4):653-658.

Isaksson, O., Kossmann, M., Bertoni, M., Eres, H., Monceaux, A., Bertoni, A., Wiseall, S., Zhang, X. (2013) Value-Driven Design - A methodology to Link Expectations to Technical Requirements in the Extended Enterprise, Proceedings of the 23rd Annual INCOSE International Symposium, Philadelphia, PA.

Johansson, C., Hicks, B., Larsson, A., Bertoni, M. 2011, “Knowledge maturity as a means to support decision making during product-service systems development projects in the aerospace sector." Project Management Journal 42(2): 32-50.

-—- 2010. Systems Engineering Handbook: A Guide for System Life Cycle Processes and Activities. Version 3.2. Revised by M. Krueger, D. Walden, and R. D. Hamelin. San Diego, CA (US): INCOSE.

Soban, D.S., Hollingsworth, P., Price, M.E. 2011. "Defining a Research Agenda in Value Driven Design: Questions That Need to be Asked." Air Transport and Operations Symposium 2011, 11p.

Takai, S. and Kalapurackal, R. S. 2012. "Sensitivity analysis of relative worth in quality function deployment matrices." Concurrent Engineering 20(3): 195-202.

Woolley, M., Scanlan, J. P., Eveson, W. 2001, “Optimising the development of a medical device using formal engineering design techniques and the coda-system." Proceedings of the 7th International Conference on Concurrent Engineering, Bremen, D. 


\section{Biography}

Marco Bertoni is Associate Professor in mechanical engineering at Blekinge Institute of Technology. His research focuses on the concept of value to complement traditional Requirements Engineering process, and encompasses engineering knowledge management approaches to support early stage decision-making in design. He has experience in European and national research projects in the domain of automotive and aerospace.

Alessandro Bertoni is a Ph.D. student in Product Innovation at the Division of Innovation and Design at Luleå University of Technology in Sweden. With a background on Management Engineering he is focusing his research on studying new methods and tools for the assessment and communication of the value of a product or service in the early stages of the design process. He is mainly working in close collaboration with the aerospace industry in the frame of the EU FP7 CRESCENDO project.

Ola Isaksson is a senior company specialist in product development at GKN Aerospace Engine Systems, Sweden, where he has been working since 1994. He is also an adjunct professor at Luleå University of Technology in Functional Product Development. He has a MSc in Mechanical Engineering, a PhD in Computer Aided Design and an associate professorship in functional product development. Ola's work and research experience concern methods, tools and processes include areas such as computational support for product development, knowledge based engineering, value based engineering, systems engineering and product-service system development. He led the Value Driven Design work in the CRESCENDO project.

Henrik Amnell is a Senior Design Engineer working for GKN Aerospace Engine Systems (formerly known as Volvo Aero). He has during his 12 years with the company worked in leading technical positions in several engine component development projects. In his current role he is part of planning and supporting technology development aiming for future engine component projects. 